\title{
La imperiosa necesidad de telemedicina en la atención de diabetes durante la pandemia de COVID-19. Un estudio de abordaje integral
}

\author{
Rubén Silva-Tinoco* y Viridiana de la Torre-Saldaña
}

Servicios de Salud Pública, Clínica Especializada en el Manejo de la Diabetes del Gobierno de la Ciudad de México, México

\section{Resumen}

Introducción: Los pacientes con diabetes experimentan dificultades para mantener el control glucémico durante el confinamiento por la pandemia de COVID-19, con el riesgo de presentar complicaciones crónicas de la diabetes y COVID-19 grave. Objetivo: El propósito de este estudio fue evaluar la conversión de un centro de atención primaria presencial de diabetes a un servicio de telemedicina por llamada telefónica. Métodos: Se realizaron consultas médicas por llamada telefónica durante la etapa inicial del confinamiento (abril a junio de 2020), para continuar el seguimiento de pacientes ingresados a un programa de atención multicomponente en diabetes. Resultados: Se realizaron 1118 consultas por llamada telefónica para continuar el seguimiento de 192 pacientes con diabetes tipo 2. Participaron diferentes profesionales de distintas áreas de la salud: atención médica, educación en diabetes, nutrición, psicología y podología. Conclusiones: La atención multicomponente en diabetes se transformó con éxito de un esquema de atención presencial a un servicio de telemedicina. Numerosos pacientes de atención primaria pueden ser candidatos a telemedicina. Se debe considerar un rediseño del modelo de atención que incorpore la telemedicina para mitigar la carga de morbimortalidad en enfermedades crónicas impuesta por la pandemia de COVID-19, pero también para la era pos-COVID-19.

PALABRAS CLAVE: Diabetes. Telemedicina. COVID-19. Cuidado en diabetes.

\section{The imperious need for telemedicine for the care of diabetes during the COVID-19 pandemic. A comprehensive approach study}

\begin{abstract}
Introduction: Patients with diabetes experience difficulties to maintain glycemic control during the confinement due to the COVID-19 pandemic, with the risk of developing diabetes chronic complications and severe COVID-19. Objective: The purpose of this study was to evaluate the conversion of an outpatient diabetes primary care center from a face-to-face care modality to a telemedicine care service by telephone. Methods: Medical consultations were made by telephone during the initial phase of confinement (April to June 2020), to then continue the follow-up of patients admitted to a multicomponent diabetes care program. Results: A total of 1,118 consultations were made by telephone and follow-up was subsequently continued in 192 patients with type 2 diabetes. Different professionals from different health areas participated, including medical care, diabetes education, nutrition, psychology and podiatry. Conclusions: Multicomponent diabetes care was successfuIly transformed from a face-to-face care modality to a telemedicine service. Many primary care patients may be candidates for telemedicine. A redesign of the care model that incorporates telemedicine should be considered to mitigate chronic diseases burden of morbidity and mortality imposed by COVID-19 pandemic, but also for the post-COVID-19 era.
\end{abstract}

KEY WORDS: Diabetes. Telemedicine. COVID-19. Diabetes care.

Correspondencia:

*Rubén Silva-Tinoco

E-mail: rosilva@sersalud.cdmx.gob.mx
Gac Med Mex. 2021;157:323-326

Disponible en PubMed

www.gacetamedicademexico.com

0016-3813/@ 2020 Academia Nacional de Medicina de México, A.C. Publicado por Permanyer. Este es un artículo open access bajo la licencia CC BY-NC-ND (http://creativecommons.org/licenses/by-nc-nd/4.0/). 


\section{Introducción}

La pandemia por la enfermedad del coronavirus 2019 (COVID-19) ha afectado desproporcionadamente a pacientes con enfermedades crónicas. ${ }^{1}$ Los pacientes con diabetes tipo 2 tienen mayor riesgo de complicaciones y mayor riesgo de muerte cuando contraen COVID-19. ${ }^{2}$ El confinamiento por esta enfermedad obstaculizó la prestación de servicios médicos generales y especializados, porque se instó a los pacientes a quedarse en casa, con lo cual quedaron sin el seguimiento médico regular. Confinados en sus hogares, los pacientes pueden cursar con desenlaces desfavorables si presentan dificultades para ejecutar conductas de autocuidado y adherencia al tratamiento de sus enfermedades. ${ }^{3,4}$

La telemedicina incluye la integración de tecnologías de comunicación para mejorar los servicios de atención médica a personas con dificultades para acudir con profesionales de la salud, quienes a su vez se han visto obligados por la pandemia de COVID-19 a encontrar formas innovadoras de tratar a los pacientes. Globalmente, durante esta pandemia se ha promovido la incorporación de la telemedicina para la atención de enfermedades no transmisibles.,

Los modelos de atención integrada multicomponente para diabetes incluyen estrategias para mejorar la calidad de la atención. ${ }^{7}$ Investigaciones anteriores han mostrado una mejora importante en el logro de los objetivos de atención de la diabetes con estas estrategias,,${ }^{7,8}$ pero la evidencia es escasa en la modalidad de telemedicina.

Como resultado de la temporalidad indefinida de la pandemia de COVID-19, que requerirá continuar con medidas para reducir el riesgo de contagio y la propagación del virus entre las personas, la identificación de estrategias en la prestación de servicios de salud que coadyuven a ello puede mitigar el impacto por la pandemia de COVID-19. El propósito de este estudio es evaluar la conversión de los servicios convencionales de atención primaria multicomponente a la modalidad de telemedicina durante la etapa inicial del confinamiento por COVID-19.

\section{Métodos}

La Clínica Especializada en el Manejo de la Diabetes del Gobierno de la Ciudad de México es un centro de atención primaria ubicado en la alcaldía de Iztapalapa. Los pacientes participan en el programa DIABEMPIC
(Diabetes EMPowerment and Improvement of Care, registrado en ClinicalTrials.gov con el identificador NCT04245267), una iniciativa diseñada para mejorar los desenlaces clínicos mediante una intervención multicomponente, que incluye atención interdisciplinaria y educación para el autocuidado. ${ }^{8,9}$ Los pacientes fueron referidos previamente de centros de salud de atención primaria, son beneficiarios del Instituto de Salud para el Bienestar (sistema público) y una alta proporción pertenece a sectores sociales económicamente desfavorecidos. ${ }^{10}$

En México, el 3 de abril de 2020 se declaró una emergencia sanitaria por la pandemia de COVID-19 y se sugirió a los sujetos mayores de 60 años y con enfermedades no transmisibles atender el confinamiento para disminuir el riesgo de contagio en este grupo vulnerable. Lo anterior condicionó una importante reducción en la prestación de servicios de atención de enfermedades crónicas por la disminución en la asistencia de los pacientes a sus citas médicas y por la asignación de recursos para la atención de la pandemia. Por lo tanto, en la Clínica Especializada en el Manejo de la Diabetes surgió la necesidad de conversión de la atención ambulatoria a la modalidad de telemedicina.

Se realizó un estudio prospectivo transversal de pacientes atendidos en las primeras 10 semanas de iniciado el servicio de telemedicina ( 20 de abril al 30 de junio de 2020). Los profesionales de la salud sin indicación de confinamiento llevaron a cabo una estrategia de consulta por llamada telefónica, cuyo contenido estaba enfocado en la atención de la diabetes. Todos los pacientes programados se incluyeron en el análisis; no hubo criterios de exclusión. Los pacientes otorgaron su consentimiento verbal para ser atendidos con telemedicina. Se procuró que los contenidos abordados estuvieran relacionados con barreras o problemas documentados en citas anteriores, para dar seguimiento individualizado mediante mensajes sencillos; se procuró no incluir nuevos contenidos. También se proporcionó información acerca de los riesgos de contagio de COVID-19 y temas respecto a la epidemia. La estrategia de equipo interdisciplinario y de manejo de casos involucró a una endocrinóloga, una nutrióloga, una enfermera educadora en diabetes, un psicólogo y una podóloga. Se procuró que no existieran cambios importantes en el tratamiento farmacológico y se sugirió una consulta presencial a quienes la requirieran. Además, en la farmacia de la clínica fue flexible el surtimiento de medicamentos y los familiares pudieron recogerlos. 
Los datos se obtuvieron de los registros de las consultas diarias. El estudio fue aprobado por la dirección médica de la institución.

\section{Resultados}

Durante las 10 semanas del estudio se llevaron a cabo 1181 consultas telefónicas a 192 pacientes, con un promedio de 6.1 consultas por paciente, distribuidas entre las cinco disciplinas de salud involucradas. La mayoría de los pacientes fue del sexo femenino (66.1\%), $50.9 \%$ tenía hipertensión y $45.8 \%$, obesidad. La edad media fue $54.1 \pm 10.8$ años y la media del tiempo de evolución de la diabetes, $11.3 \pm 7.7$ años.

En la Tabla 1 se presentan las características clínicas de la población de estudio, los servicios telefónicos proporcionados y los recursos tecnológicos a los que tenían acceso los pacientes: $98.4 \%$ a teléfono, $73.4 \%$ a computadora o teléfono inteligente con función de videochat y $69.7 \%$ a internet. En la farmacia de la clínica se suministraron 263 recetas de medicamentos. Una alta proporción de pacientes consideró útil esta estrategia de telemedicina para el manejo de su enfermedad (95\%).

\section{Discusión}

Este estudio exploró la transformación de un modelo de atención presencial interdisciplinaria de diabetes en un modelo de telemedicina vía telefónica, dentro del primer nivel de atención. La conversión fue exitosa e incluyó una estrategia de consultas de seguimiento interdisciplinario y el suministro de medicamentos, con la intención de dar continuidad a la atención de pacientes con diabetes en un momento temprano del confinamiento por COVID-19 en México. Cabe destacar que una proporción importante de pacientes refirió la falta de acceso a internet. Los resultados permiten proponer estrategias para la conversión de los servicios de atención convencional de enfermedades no transmisibles en servicios de telemedicina; además, sugieren la oportunidad de continuar con esquemas de atención híbridos (presencial y telemedicina) acordes a las necesidades de los pacientes y a la evolución de la pandemia.

Hasta donde tenemos conocimiento, este es el primer estudio acerca de la conversión de atención multidisciplinaria de diabetes en atención de telemedicina a raíz de la pandemia de COVID-19.
Tabla 1. Características clínicas de los participantes del estudio y tipos de consulta telefónica en la estrategia de telemedicina para atención de la diabetes $(n=192)$

\begin{tabular}{|c|c|c|}
\hline Características & \multicolumn{2}{|c|}{ Media $\pm \mathrm{DE}$} \\
\hline Edad en años & \multicolumn{2}{|c|}{$54.1 \pm 10.8$} \\
\hline Duración de la diabetes (años) & \multicolumn{2}{|c|}{$11.3 \pm 7.7$} \\
\hline Edad al diagnóstico (años) & \multicolumn{2}{|c|}{$43.4 \pm 10.2$} \\
\hline & $\mathrm{n}$ & $\%$ \\
\hline Sexo femenino & 127 & 66.1 \\
\hline Educación primaria o menos & 88 & 45.8 \\
\hline Hipertensión & 106 & 50.9 \\
\hline Obesidad & 88 & 45.8 \\
\hline Tratamiento con insulina & 61.5 & 128 \\
\hline Acceso a teléfono & 189 & 98.4 \\
\hline Acceso a internet & 134 & 69.7 \\
\hline $\begin{array}{l}\text { Acceso a computadora/teléfono inteligente con } \\
\text { videochat }\end{array}$ & 141 & 73.4 \\
\hline $\begin{array}{l}\text { Percepción de la utilidad de la atención por } \\
\text { telemedicina para el manejo de enfermedades }\end{array}$ & 183 & 95.3 \\
\hline Consultas de telemedicina vía telefónica & 1181 & 100 \\
\hline Consulta médica & 465 & 39.3 \\
\hline Psicología & 248 & 20.9 \\
\hline Educación en diabetes & 232 & 19.6 \\
\hline Podología & 200 & 16.9 \\
\hline Nutrición & 36 & 3.2 \\
\hline
\end{tabular}

Alromaihi et al. informaron sobre la implementación exitosa de telemedicina con intercambio de mensajes de texto y videollamadas; ${ }^{11}$ en nuestro estudio, la atención por un equipo interdisciplinario y el suministro de medicamentos fueron incorporados de forma adicional.

En un reporte previo en México, los pacientes disminuyeron las actividades de autocuidado debido a las dificultades para ejecutarlas durante el confinamiento por la pandemia de COVID-19. ${ }^{4}$ Existe evidencia de que los pacientes con diabetes experimentan una mayor gravedad de COVID-19, que puede acentuarse en aquellos con control glucémico deficiente..$^{12,13}$ Por lo tanto, es una necesidad optimizar la atención de la diabetes, tanto por el impacto que puede tener en la pandemia de COVID-19 como por la implicación de la carga en la salud pública que la diabetes puede tener en la era pos-COVID-19.

Algunas consideraciones deben ser analizadas antes de implementar estrategias de atención de 
telemedicina, como la presencia de algunos determinantes de la salud y el acceso a recursos tecnológicos, ya que la población con desventajas sociales podría enfrentar barreras para integrarse a esta modalidad de atención, como se evidencia en este estudio. Por lo anterior, las intervenciones de telemedicina deben adaptarse a estas barreras y dificultades específicas impuestas por el confinamiento por COVID-19 en los pacientes con diabetes mellitus tipo 2. Nuestros hallazgos respaldan una propuesta alentadora para abordar esas barreras. Es probable que la telemedicina se convierta en una parte integral de la prestación de la atención médica, especialmente en las enfermedades crónicas, pero las visitas presenciales continuarán y el desarrollo de modelos híbridos surge como una respuesta sólida, incluso en la era pos-COVID-19. Sin embargo, pronto se debe plantear un marco regulatorio y el establecimiento de protocolos para garantizar la calidad de la atención y la seguridad del paciente.

\section{Conclusiones}

Los servicios multicomponente presenciales de atención en diabetes se transformaron con éxito a la modalidad de telemedicina. En el primer nivel de atención de salud, numerosos pacientes con diabetes mellitus tipo 2 pueden ser candidatos para integrarse a este y otros tipos de estrategias de telemedicina en escenarios de contingencias sanitarias. Se debe considerar un rediseño del modelo de atención con la incorporación de tecnologías y la telemedicina, para mitigar la morbimortalidad por enfermedades crónicas durante la pandemia de COVID-19, así como en la era pos-COVID-19.

\section{Conflicto de intereses}

Los autores declaran que no tienen conflictos de interés.

\section{Financiamiento}

No se dispuso de financiación pública o privada para esta investigación.

\section{Responsabilidades éticas}

Protección de personas y animales. Los autores declaran que para esta investigación no se realizaron experimentos en seres humanos ni en animales.

Confidencialidad de los datos. Los autores declaran que siguieron los protocolos de su centro de trabajo sobre la publicación de datos de pacientes.

Derecho a la privacidad y consentimiento informado. Los autores declaran que en este artículo no aparecen datos de pacientes.

\section{Bibliografía}

1. Wu Z, McGoogan JM. Characteristics of and important lessons from the coronavirus disease 2019 (COVID-19) outbreak in China: summary of a report of 72314 cases from the Chinese Center for Disease Control and Prevention. JAMA. 2020;323(13):1239-1242.

2. Hernández-Galdamez DR, González-Block MÁ, Romo-Dueñas DK, Lima-Morales R, Hernández-Vicemte IA, Lumbreras-Guzmán M, et al. Increased risk of hospitalization and death in patients with COVID-19 and pre-existing noncommunicable diseases and modifiable risk factors in Mexico. Arch Med Res. 2020;51:683-689.

3. Banerjee M, Chakraborty S, Pal R. Diabetes self-management amid COVID-19 pandemic. Diabetes Metab Syndr. 2020;14:351-354.

4. Silva-Tinoco R, González-Cantú $A$, de la Torre-Saldaña V Guzmán-Olvera E, Cuatecontzi-Xochitiotzi T, Martínez.Castillo L, et al. Effect in self-care behavior and difficulties in coping with diabetes during the COVID-19 pandemic. Rev Mex Endocrinol Metab Nutr. 2020;8:13-19.

5. Faradji-Hazán RN, Garnica-Cuéllar JC, Hernández-Arizpe LM, LeónSuárez A, Mancillas-Adame L, Márquez-Rodríguez E, et al. Recomendaciones de la Sociedad Mexicana de Endocrinología SMNE ante el COVID-19 para el tratamiento de personas que viven con DM2. Rev Mex Endocrinol Metab Nutr. 2020;7:4395.

6. Hartmann-Boyce J, Morris E, Goyder C, Kinton J, Perring J, Nunan D, et al. Diabetes and COVID-19: risks, management, and learnings from other national disasters. Diabetes Care. 2020;43:1695-1703.

7. Lim LL, Lau ESH, Kong APS, Davies MJ, Levitt NS, Eliasson B, et al. Aspects of multicomponent integrated care promote sustained improvement in surrogate clinical outcomes: a systematic review and meta-analysis. Diabetes Care. 2018;41(6):1312-20.

8. Silva-Tinoco R, Cuatecontzi-Xochitiotzi $T$, de la Torre-Saldaña V, León-García E, Serna-AlvaradoJ, Guzmpan-Olvera E, et al. Role of social and other determinants of health in the effect of a multicomponent integrated care strategy on type 2 diabetes mellitus. Int J Equity Health. 2020;19:75

9. Silva-Tinoco R, Cuatecontzi ETC, De la Torre-Saldaña V, et al. 1178-P: multicomponent integrated care program benefits on cardiovascular risk factors for adults with diabetes and high cardiovascular risk: the DIABEMPIC Program. Diabetes. 2020;69:1178-P.

10. Silva-Tinoco $R$, Cuatecontzi-Xochitiotzi $T$, de la Torre-Saldaña $V$, León-García E, Serna-Alvarado J, Orea-Tejeda A, et al. Influence of social determinants, diabetes knowledge, health behaviors, and glycemic control in type 2 diabetes: an analysis from real-world evidence. BMC Endocr Disord. 2020;20:130.

11. Alromaihi D, Alamuddin N, George S. Sustainable diabetes care services during COVID-19 pandemic. Diabetes Res Clin Pract. 2020;166:108298.

12. Apicella M, Campopiano MC, Mantuano M, Mazoni L, Coppelli A, del Prato S, et al. COVID-19 in people with diabetes: understanding the reasons for worse outcomes. Lancet Diabetes Endocrinol. 2020;8:782-792.

13. Zhu L, She Z-G, Cheng X, Quin JJ, Zhang XJ, Cai J, et al. Association of blood glucose control and outcomes in patients with COVID-19 and pre-existing type 2 diabetes. Cell Metab. 2020;31:1068-1077. 